\title{
Remote Triggered Analog Communication Laboratory for E-Learning
}

\author{
http://dx.doi.org/10.3991/ijoe.v9iS5.2773 \\ C.M. Markan ${ }^{1}$, Goutam Kumar ${ }^{1}$, Sajal Mittal ${ }^{2}$, Priti Gupta ${ }^{1}$, \\ Satendra Gupta ${ }^{1}$, Anshul Satsangi ${ }^{1}$, Ashima Gupta ${ }^{1}$, Garmima Kapur ${ }^{1}$ \\ ${ }^{1}$ Dayalbagh Educational Institute, Dayalbagh, Agra, India \\ ${ }^{2}$ Indian Institute of Technology, Kanpur, India
}

\begin{abstract}
Currently most universities have Online-learning environments ready to be remotely accessed through the internet. Remote labs have emerged as a viable alternative for developing skills and to learn how to deal with laboratory instruments in the absence of real labs. Remote labs can provide remote access to hardware and simulators, and can allow students to perform experiments without time and location restrictions. In addition, they provide the necessary guidance and also constrain user operation in order to avoid dangerous situations (both from set-up integrity and from the user's point of view).In this paper, we describe a hierarchical model of a remote lab for analog communication that is focused for undergraduate and engineering students. The lab brings together a two level learning approach. i). A basic device level approach for new users and ii). An advanced block level approach for advanced users. Features like ondemand service, virtual breadboard, interactive instrument panels and supporting supplementary materials, makes our lab the right candidate to be used in e-learning. The lab is currently being used for e-learning at distance education centers of the university.
\end{abstract}

Index Terms-Remote Laboratory, Analog communication, e-Learning

\section{INTRODUCTION}

e-Learning or computer-based remote learning is a potential solution to the knowledge crisis in our society. In order to acquire practical competence in engineering education, experimentation on actual equipment in labs is important and in the case of e-learning it becomes all the more necessary. The most attractive feature of e-learning is that it is student-centered. It accommodates individual preferences and needs and empowers students of various backgrounds to have equal access to the best resources and referral material, lecture sessions, tutoring [1].

Today, e-learning offers a variety of services such as building and designing training courses, offering webbased programs for learning and content management [2].The change in education system and the increase in the knowledge requirement have created significant changes inthe way the learning process takes place. These days, there is a constant need to improve our skills, to keep our knowledge up-to-date and to obtain knowledge on new topics - especially in the case of higher level studies, where technology is constantly evolving [3].

Despite all the advances in e-learning, it is still deficit in providing an essential aspect of engineering i.e. laboratory education [4-5]. Development of remote labs is an important step towards providing a complete knowledge framework to the students and enrichment of society. Remote labs are envisaged as tele-operation of real laboratories, since it allows students to obtain practical knowledge without the space and time constraints of classical face-to face education.

There has been considerable growth in the area of Remote labs in the past decade. Remote labs have been developed in almost all the streams of education e.g. robotics, chemical, mechanical, electronic etc [6-8]. Some of these labs are designed to be used for complex tasks and require a lot of prior knowledge [11] of the subject and practice from the student's end. Hence, it becomes difficult for an average student to work on these labs in the absence of any guidance. It is necessary to convey these complex experiments in a more familiar manner, so that they are understood by even a sophomore student [9]. This opens up a need to develop a hierarchical model of these kind of labs that can be utilized equally well by average as well as above average students. This demands that the structure and the architecture of remote labs should be inspired from fundamental educational system models [10].

Analog communication is an important subject in engineering. It covers the current and advanced concepts of analog communication that have been implemented in modern world applications. A Block diagram approach is mostly adopted in this regard [12]. Some students find it difficult to understand the block diagram approach. Therefore, providing analog communication experiments in remote environment requires a more sophisticated mechanism so that it is easily understood by all students.

Several labs have been proposed for analog communication that follows the block diagram approach. However, none of them talks about a hierarchical model and design, that provides knowledge, application, synthesis and evaluation to the students at the same time. These steps are quite essential for remote labs to function properly in an e-learning environment. Analog Communication experiments like modulation, demodulation etc. are traditionally conducted using a breadboard and electronic components. We have used Emona DATEx board (Plug-in module for NI-ELVIS) to provide access to analog communication [16], we have introduced a methodology where these experiments can be experiments. To provide access to communication experiments using Device Level approach, we have introduced a methodology where these experiments can be performed using analog devices e.g. registers, capacitors and inductors. 


\section{SPECIAL FOCUS PAPER \\ REMOTE TRIGGERED ANALOG COMMUNICATION LABORATORY FOR E-LEARNING}

Another feature of these laboratories is Multi-user scalability [9]. A major limitation faced by the remote laboratories today is scalability. Since the methodology used to convert a real lab to remote lab is usually simply automating the existing experiment without any regard to exploiting the technology for scalability, this ensures that the limitations faced by real labs i.e. 'one user at a time' also become a limiting factor for remote labs. In our lab, because of the multi-user scalability introduced, multiple users working on the lab get an 'illusion of parallelism' since they get their results 'on demand' without any requirement for booking slots for exclusive access to instruments. In this paper in section II we discuss the hierarchical model for analog communication remote lab. In section III, we describe the architectural details, hardware implementation and software design and GUI of the innovative methodology. In section IV, we discuss about our mathematical approach using an example experiment and comparison of experimental results between the two approaches. In section $\mathrm{V}$, we discuss about the deployment of these labs in e-learning environment. In section VI, we point out some of the current and future challenges with remote labs development

\section{ANALOG COMMUNICATION HIERARCHICAL MODEL}

Every step towards the development and design of any technology needs inspiration and motivation from past work and futuristic imaginations. Every system has to adapt basic rules initially before building itself complex and to be used for more complicated tasks. Best example for this is the human brain that develops itself in a hierarchical manner, from first cortical layer to the sixth layer; every layer encompasses an additional dimension, helping in understanding complex tasks. Blooms taxonomy is an example for classification of educational learning objectives. Its goal is to focus on affective, cognitive and practical improvements of students. We have modulated six levels of blooms taxonomy in remote labs perspective.

1. Knowledge: This level is the lowest level of thinking requiring the least cognitive effort. It is defined as the remembering concepts learned in the past.

2. Comprehension: This level focuses on students' ability to comprehend new information presented to them and translate the information from one form into another.

3. Application: This level requires students to use the new knowledge that was learnt in new or different situations, such as in a simulated or real workplace setting.

4. Analysis: This level develops creative skills within the students as it requires to compare their outcomes with other possible outcomes.

5. Synthesis: This level is often seen as the opposite of Analysis. It involves the capability to assemble individual components to create a new design.

6. Evaluation: This level of thinking requires students to evaluate or review the value or relative worth of ideas or objects based on predetermined criteria.

In this model, educational objectives of a system have been discussed. We have followed this design to derive analog communication remote lab. We have argued two different approaches in this context.

a). Block Diagram Approach: in this approach, ana$\log$ communication concepts are learned using mathemat-

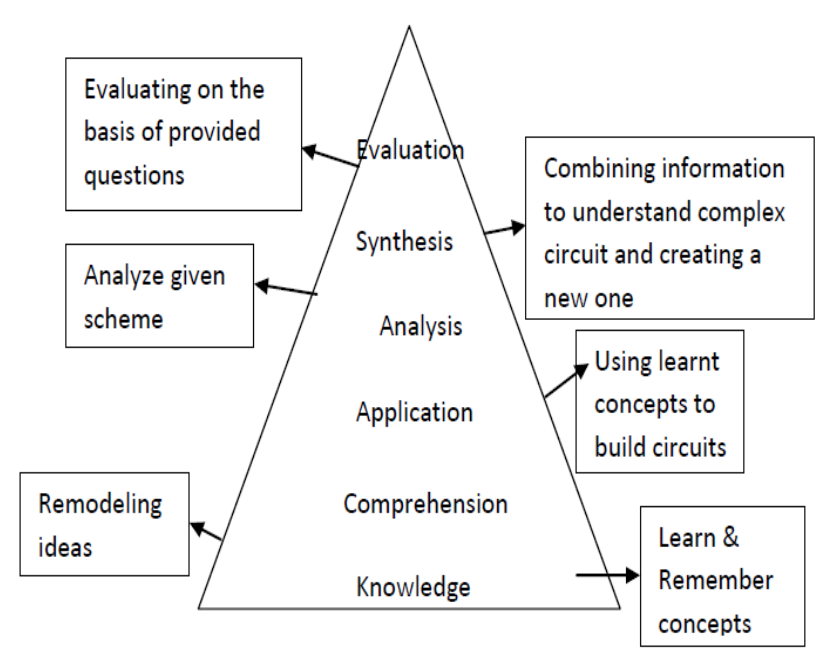

Figure 1. Pyramid depicts various levels of thinking that we can implement

ical equations where students can easily implement theoretical concepts in labs by using block diagram approach and used as standard notations in text books to describe mathematical equations. This approach is easier for the higher level students and for designing complex circuits. We have used NI ELVIS II and Emona DATEx in this context. DATEx board consists all the required blocks to perform analog communication experiments.

b). Device Level Approach: This approach follows traditional components e.g. registers, capacitors etc. Students can utilize these components in building up communication experiments as they learnt in their class. This is more fundamental type of approach that integrates the methodologies for both higher level and lower grade students. We have used LXI instruments to develop communication remote lab in device level approach that delivers these experiments not only in traditional 'one-equipmentone-user' mode but also provides multi-user scalability.

\section{IMPLEMENTATION}

\section{A. Lab Architecture}

Client-Server Architecture [13, 18] as shown in Figure2 describes the design for block diagram approach using ELVIS DATEx bundle and more common device level approach based on LXI methodology. This architecture provides a mechanism to build scalable model for these approaches that is nowhere else found in state of the art remote labs literature. It also eliminates the requirement of LabVIEW runtime engine on client's end. This architecture classifies two different mechanisms for device level and block level approaches. It utilizes virtualization and cloud computing concepts to design a scalable model of these labs. Queuing mechanism has been implemented that pipelines the user requests in FIFO manner. In interactive mode, client has to book a slot and owns the complete hardware control. However, in batch mode client's don't have access to real instrument panels. For this purpose, interactive panels are designed that looks like the real instrument panels and provides illusion of parallelism. These panels have buttons and graphics that looks like the clone of real panel and gives students a feel of a traditional instrument.

Remote labs utilize high end instrumentation in their development, which costs much more than traditional lab 


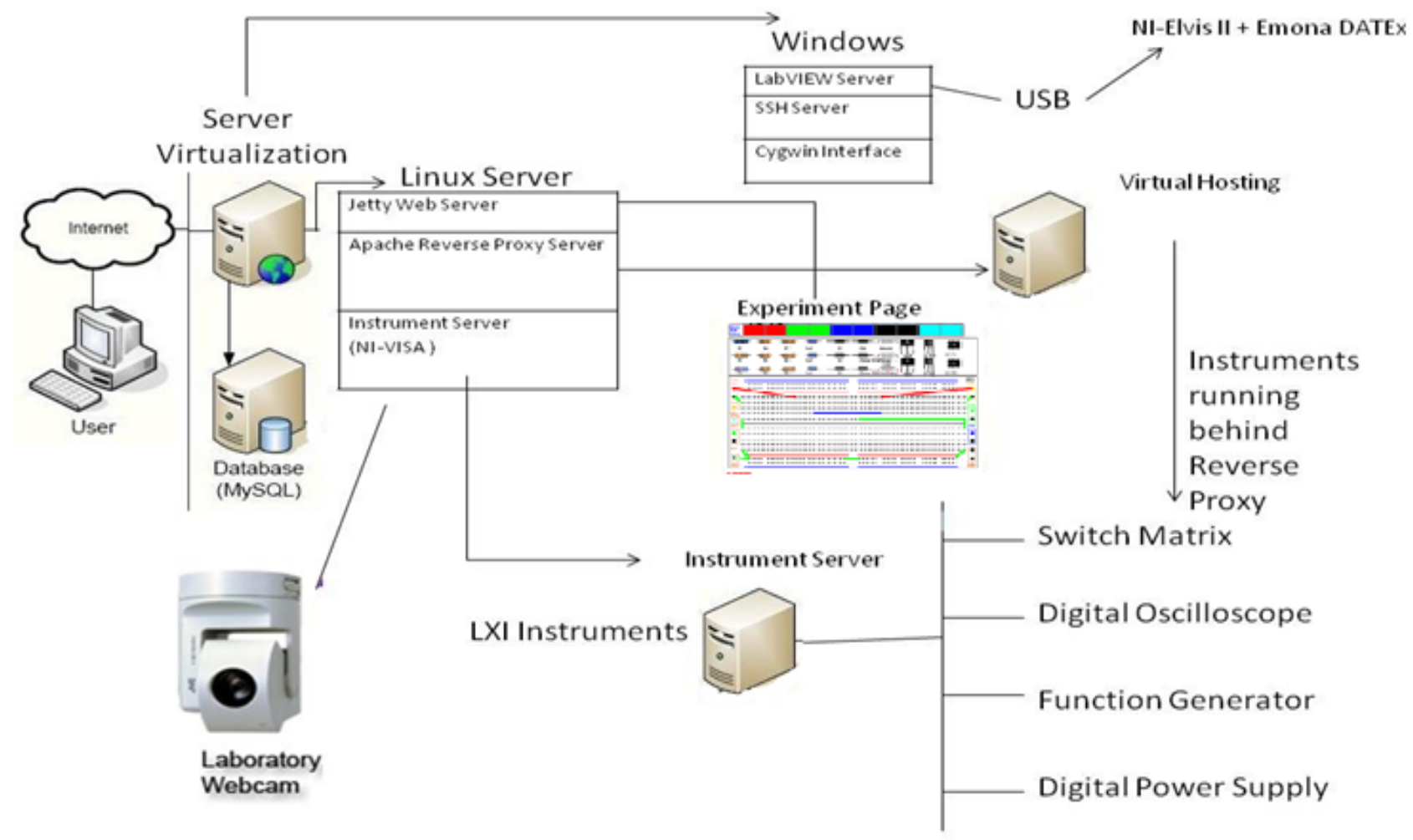

Figure 2. Remote lab architecture including instrument protocols and servers for analog communication labs elaborating use of virtualized servers and data flow mechanism

instruments. Now the challenge remains the scalability of these labs to justify their development cost. In this context, iLabs architecture [15] has been developed which is a three tiered model, consisting of lab clients, service broker and lab servers. However, this architecture is only valid for batched experiments. Sahara [19] provides a common integrated platform for remote laboratories to be accessed and shared among institutions. Cloud computing can be used as a solution of handling multiple-users and resource sharing in client/server architecture. Since, it provides a promising approach to overcome some of the drawbacks of client/server architecture. Due to its elasticity, cloud computing paradigm is suitable to solve the problem of scalability in remote labs. Cloud computing follows the utility computing model of pay-per-rent model allowing users to pay for the services they actually use

\section{B. Hardware Design}

In order to perform communications experiments in a remote environment, there is a need for eliminating the user interface needed to perform the required connections and to provide extendibility to wiring circuits from clientside, which is accomplished by using Agilent LXI switching matrix. Figure 3. depicts the hardware setup of DATEx board.

\section{1) Relay Matrix}

Wiring up an arbitrary circuit sitting at a remote location requires configuring a interface, a switching process that can work in backend controlling wired components. Switching matrix has been utilized for this purpose, it eliminates the need to wire up user defined circuit on server side in remote lab. It is basically a LXI switch matrix device. Typically it has 8 rows by 64 columns $(8 \times 64)$ or a similar combination. The devices to be used in experiments along with instruments are put on the columns. Rows and columns form a grid and each point on the grid (row, column) has a relay. The rows are not connected to the columns but any point can be shorted by closing its relay. Thus by closing specific relays, selected devices can be connected in any desired topology, thus matching the circuit to be drawn. Information about the devices and their placements on the columns is recorded in a configuration file. This configuration file contains information about the device, its parameters and the column numbers where the device is connected. Table given below shows the sample netlist file.

TABLE I.

SAMPLE NETLIST FILE, DESCRIBING THE ROWS AND COLUMN PARAMETERS

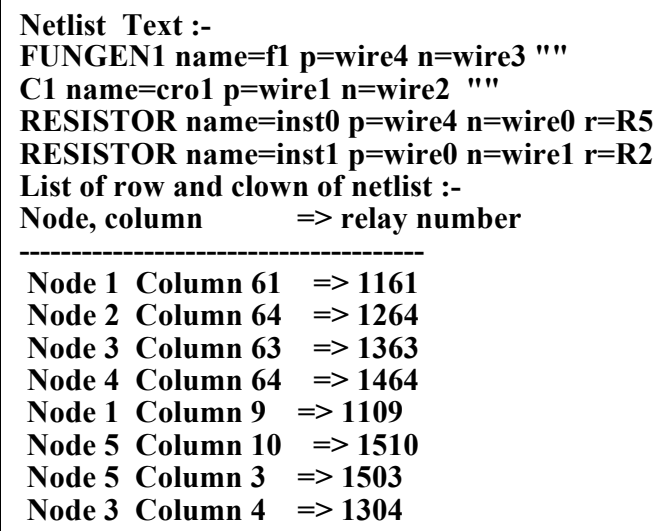




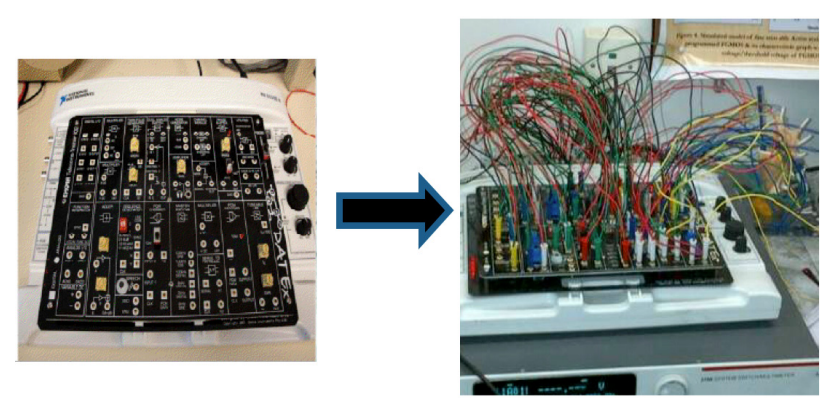

Figure 3. DATEx hardware setup of analog communication lab

\section{2) Relay configuration file:}

Each relay in the relay matrix is recognized by a relayId. This implies each (row, column) point corresponds to a relayId. The relay configuration file contains a mapping for each (row, column) to its relayId. The relay configuration file helps the system to remain independent of the underlying relay matrix hardware. If the hardware changes or the relay mapping changes, only relay configuration needs to be updated.

\section{3) Board configuration file:}

Information about the devices and their placements on the columns is recorded in a configuration file. The board configuration file contains information about the device, its parameters (e.g., $10 \mathrm{~K}$ for resistor), and the column numbers where the device is connected.

To develop remote lab for block diagram approach using DATEx bundle, Cygwin interface has been created, that communicates between Web Server and LabVIEW server using SSH protocol. LabVIEW VI's are converted into DLL's (Dynamic Link Library) that executes on Microsoft Visual Studio. DLL functions have been created inside MS visual studio for functioning of the ELVIS VI's. LabVIEW server resides behind the reverse proxy server to protect instruments from outside attacks.

To develop remote lab for device level approach, LXI methodology has been adopted. That provides several in built features i.e. web server, standard communication drivers etc. The set of equipment available for a remote user are

(i) Digital Storage Oscilloscopes

(ii) Arbitrary Waveform Generators

(iii) Spectrum Analyzers

(iv) Programmable Power supplies

(v) Programmable Variable Resistances

(vi) Digital Multimeter

These equipment use state of the art technology called LXI (LAN extension for Instrumentation) that makes it possible to use the full capacity of the instrument through internet access both in terms of instrument control but also in the form of observing the outcome in real time [14]. The use of such equipment not only a gives a student access to an experiment but also trains him/her to state of the art of instrument protocols which are rarely available to a sophomore.

Students are provided a real breadboard like screen where they can wire up any arbitrary circuit. Netlist file is generated consisting of circuit and instrument parameters that are decoded on the server. Instruments are communicated through SCPI C commands executed through command line. Parameters are sent to the instruments along with these commands that execute, and send back the results to the web server within a second. All the responses are then sent to the client through HTTP server protocol using applet-server URL data transfer. Stress test was performed to observe the total hardware execution time using this mechanism which was found around one second.

\section{Software Design}

\section{1) Virtualization Software}

Software utilities adopted to build remote labs are equally important as hardware. All the virtualization software is done in HTML, JavaScript, Java, and LabVIEW and in Perl language. Remote lab workspace has been designed using java applet. The HTML, JavaScript and the Java reside in the Web server and the Perl language compiler resides in the controller portion. The Web-based software, written in HTML, JavaScript, and Java, integrates the virtual breadboard, components, and instruments. SCPI C commands are used to communicate between server and instruments. MVC (model view controller) architecture has been implemented that separates code designed for client side request and server side processing. LabVIEW customized soft panels are designed for NI ELVIS. To provide accessibility to DATEx blocks, soft panel has been designed that consist all the controllable knobs given on the DATEx board. To display acquired data from oscilloscope, animated version of oscilloscope has been designed that makes no difference from real view. Figure 4 shows the animated designs of oscilloscope and DATEx controls. Function generator soft panel has also been designed to select waveforms and parameters from client browser.

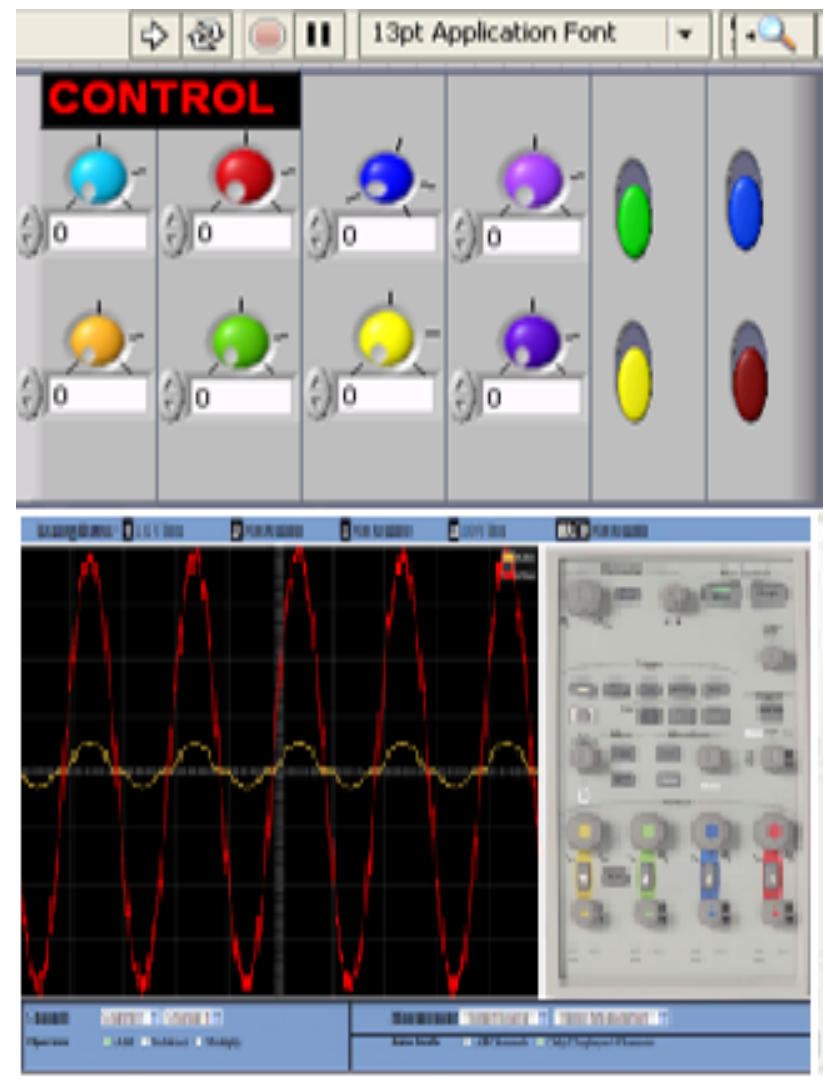

Figure 4. Upper panel shows the DATEx knobs and lower panels is for animated oscilloscope 


\section{2) Virtual Breadboard}

Sitting at a remote location it may not be possible for students to manipulate the components and wire a circuit with their fingers in the remote laboratory, however, using these labs one can operate a type of circuit-wiring tool, i.e. a relay switching matrix, to physically wire up electronics circuits at the host laboratory site using Internet access and by means of a conventional lookalike "virtual breadboard" GUI on the Web. Figure 5 shows a typical Webbased "virtual breadboard" for device based remote lab and Figure 6 shows user interface for block based remote lab, which is a photograph of a real breadboard. The component box at the top of the breadboard shows the set of electronic components available in the selected lab session.

The user is able to drag and drop any components over the virtual breadboard as desired to accomplish the necessary wiring. When the user completes the circuit and presses a "run" button, the software at the host analyzes the circuit to extract a net-list i.e. determines which hardware pins are connected together. The pin connections represent nodes. The circuit may be wired (correctly or incorrectly) on any portion of the virtual breadboard on the Web but the software turns on only the appropriate relays to connect the circuit wired on the virtual breadboard.

Remote labs web-page appears like a dashboard that shows a summarized view of all involved instruments and a virtual breadboard. The representative buttons provide a way to open up elaborate front panels of the measurement instruments, while the display panels of these instruments

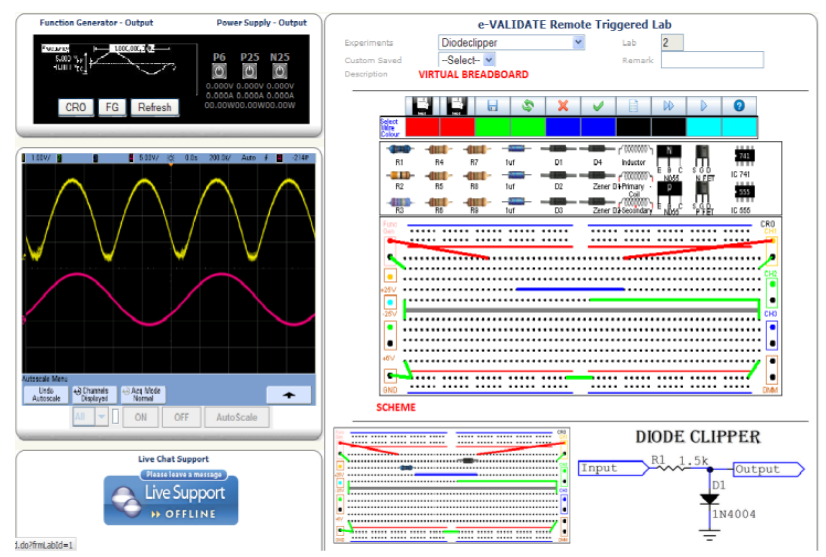

Figure 5. Front page of device level communication remote lab

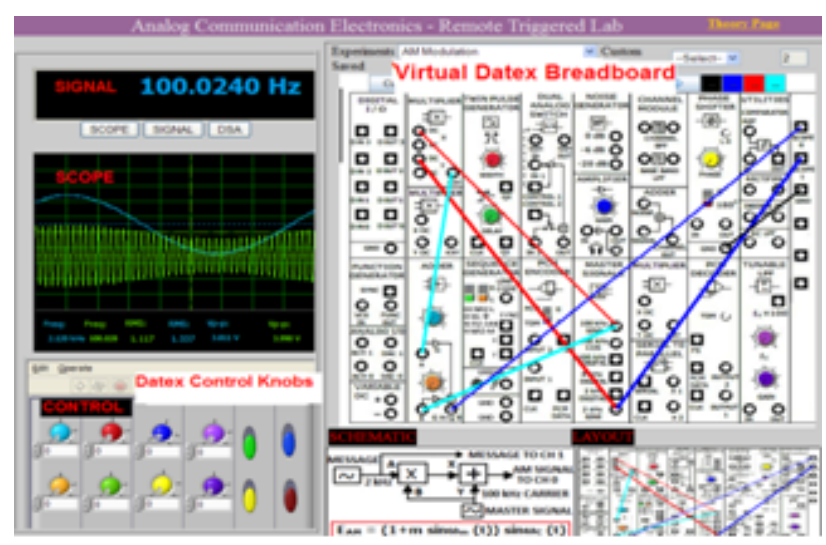

Figure 6. Front page of block level communication remote lab convey essential information for quick view. Background software ensures electrical rules and breadboard topology are encoded so as to extract an electrical circuit from the virtual breadboard GUI. Helpful buttons below the breadboard provide the facilities for saving a wired breadboard, loading a stored circuit, and running a circuit on real hardware. The interface is greatly simplified by using a graphical interface to allow the students to experience the frustrations and hands-on experiences of a real-world laboratory environment. A student trained on these virtual panels is not likely to feel out of place in a real laboratory.

\section{AMPLITUDE MODULATION EXAMPLE}

Amplitude modulation is most commonly used in electronics and communication. We will illustrate amplitude modulation experiment using device level approach as well as more specific block diagram approach. Figure 7 shows the diagram for amplitude modulation using device level and block diagram approach.

From this diagram, we have shown how we can synthesize a device level experiment design and compare it with block level experiment design. The block diagram for analog communication is little different that is traditionally used as shown in figure 8. In this example we have replaced multiplier block with rectifier and band pass filter so that the two diagrams becomes irreversible to each other. After this we measured the results with this new circuit diagram that we found similar to the results of traditional amplitude modulation circuit diagram.

Here we have demonstrated this example to elaborate the basic concept behind amplitude modulation, so that it becomes easy for any student to understand the concept when he/she goes through the block diagram of any specific experiment. This kind of approach enhances students creative skills and evaluating the concepts that he/she has

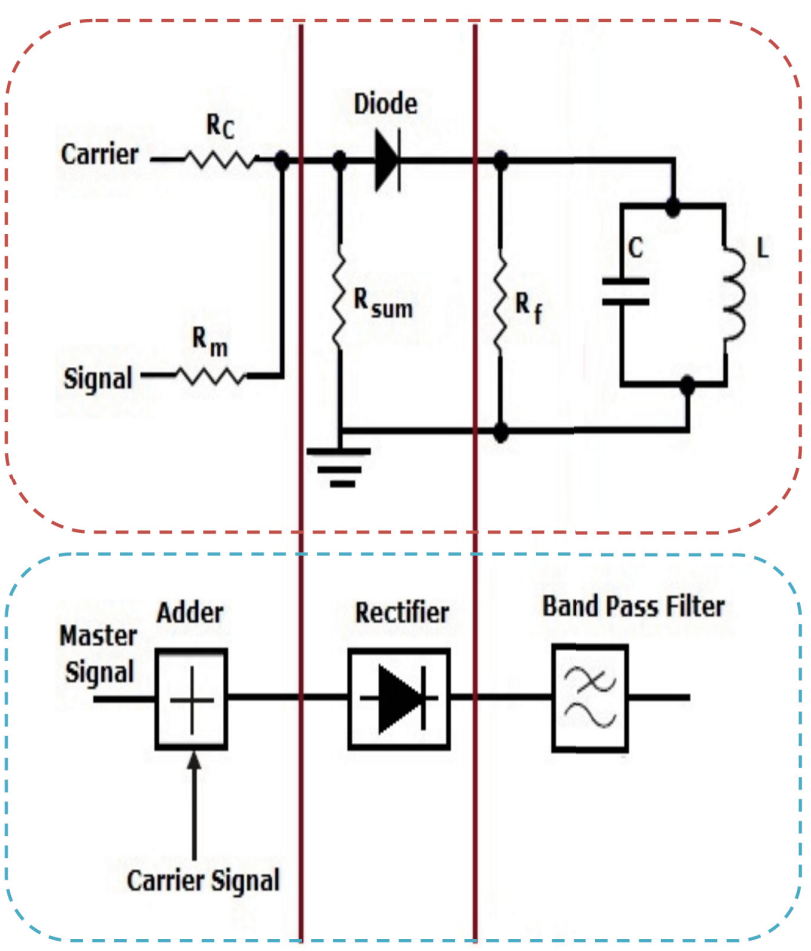

Figure 7. Synthesized diagram for amplitude modulation on device level and block level, block level diagram has been recreated to compare with the device based diagram 


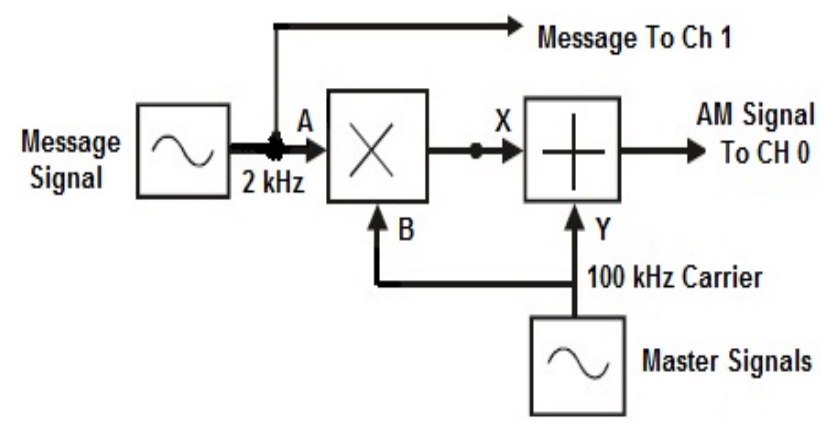

Figure 8. Traditional circuit block diagram of amplitude modulation
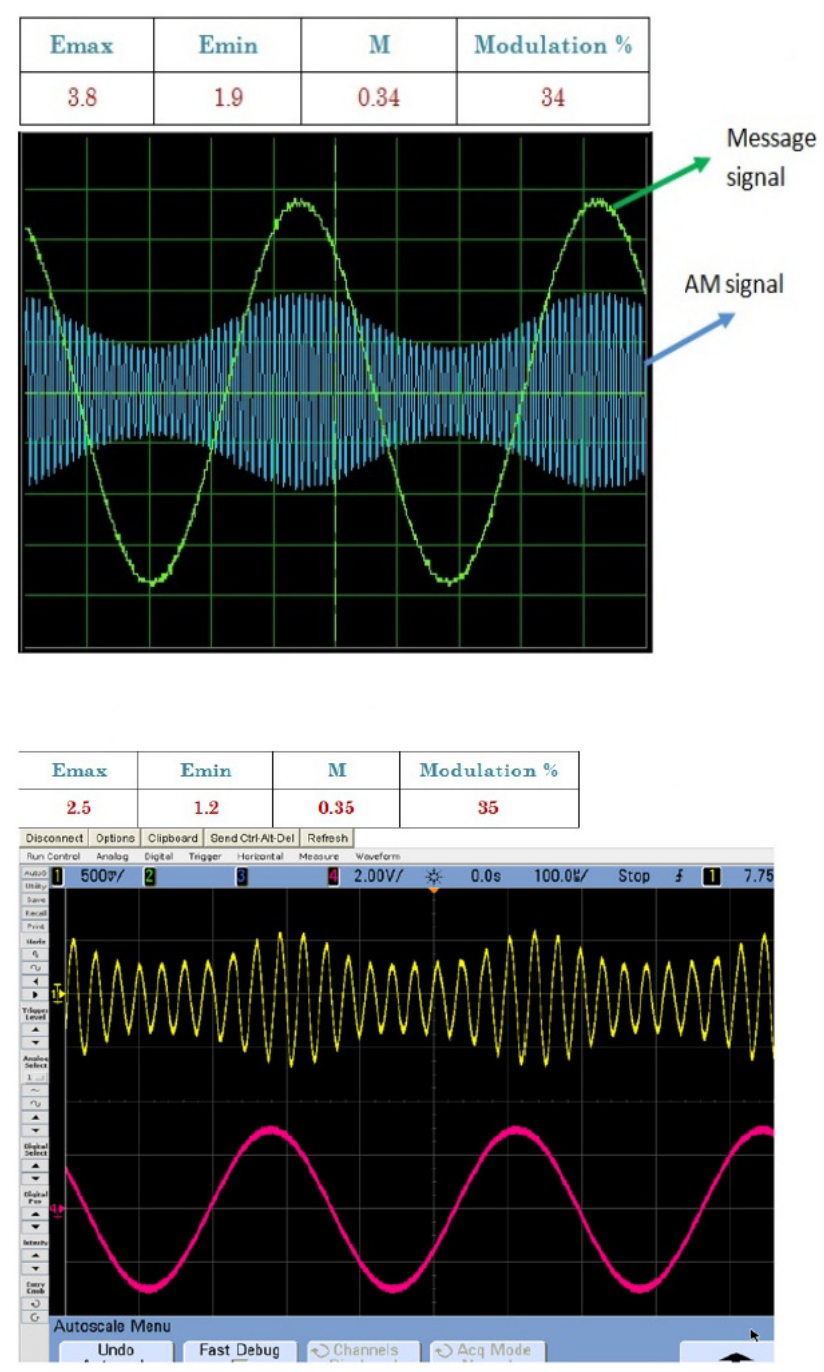

Figure 9. Results of modulation percentage displayed on oscilloscope screens

learnt in class. In short these two approaches together builds up a framework that helps to understand the basic fundamentals in an easier manner behind any complex system.

To verify the output results of the experiment, we performed amplitude modulation on both remote labs. We calculated modulation percentage in both scenarios using:

$$
\text { Modulation Percentage }=\frac{E_{\max }-E_{\min }}{E_{\max }+E_{\min }} \times 100 \%
$$

We got the following results as shown in figure 9 for both scenarios that found to be almost same. Modulation index in case of block level was measured around 34\% and in device level was computed around 35\%.

\section{DEPLOYMENT AND FIELD TESTING}

To test the feasibility of these labs in distance learning, we chose to deliver these remote labs facility to the students of distanced located centers of the university. Remote labs are scheduled in their curricula and students get 24 hour access to these labs. Initially, training session was conducted for students to provide an idea of accessing remote labs. Students were given all prior knowledge required to work on remote labs. After that, every student was asked to perform experiment from his/her interest. In our initial study we found students very comfortable with the design of remote labs that we have developed. Because the user interface designed generally [12] for remote labs quite differs from real labs. Another benefit that we observed was multiple students can perform their own experiment simultaneously unlike the real lab where the faculty demonstrates on hardware and students have to wait until the previous one finishes. Students were also agreed with the accuracy of results that they got on remote labs. We also conducted final viva-voice session using remote labs and video conferencing to test the feasibility of these labs in conducting practical examination. External examination was conducted for the students studying in distance learning and initial reports are found to be favorable in this context.

\section{CHALLENGES \& INNOVATIONS}

Early development of remote labs was confronted with several technological hurdles. Initially the challenge was the programmability and optimization of these labs. To be able to provide close to real life experience remotely was the main objective. Now we see, remote labs utilize high end instrumentation that can be accessed from anywhere. Now we see remote labs being developed for almost every field in education from electronics to robotics and mechanics. Development of these labs has certainly been advantageous to students and universities. However these labs still face few challenges. Some of the challenges are:

1. Scalability: Most remote labs are built to handle one student at a time. Therefore they need slot booking of typically $1 \mathrm{hr}$ per slot. If a student needs to do 10 labs a semester then every student needs one day of $10 \mathrm{hrs}$ in 6 months. That implies at most 150 students can perform experiments using remote labs in every semester. This is not very promising given the fact that remote labs cost nearly 10-100 times more than a real lab.

2. Communication protocols: Information exchanged between devices on a network is governed by set of rules and conventions. Different devices may follow different protoclos for exchanging information and data retrieval. These devices have to communicate with each other using shared transmission media. This transmission depends on different hardware and operating systems. So there is need to evolve some standard communication protocols for remote labs that work well in heterogeneous environment..

3. Maintanence: Another challenge with remote labs is their maintanence. Once a remote lab has been developed and deployed successfully, it demands engineers and experts to troubleshoot errors and issues that occur at running time. This is because remote 


\section{SPECIAL FOCUS PAPER \\ REMOTE TRIGGERED ANALOG COMMUNICATION LABORATORY FOR E-LEARNING}

labs utilize standard instruments that require calibration from time to time. Also, they need to be maintained because they use expensive equipment damage to whichhave huge cost implication to the host institution.

In addition to the existing benefits that remote labs provide, some innovations, if done, could further enhance their utility and accessibility. Some of these innovations include:

1. Developing Collaborative Frameworks: With an immense growth in remote labs and universities investing huge amount of money on virtualization of laboratories, developing collaborative frameworks would be a great advantage. These will provide sharing of resources between different institutions and will help in cost reduction in providing remote lab services.

2. Integration with LMS: Remote labs support a student-centric approach and provide students an environment where they can freely apply theoretical conceptsto practical use. If there is some sort of supplementary material provided with remote labs, students can utilize it to learn new theoretical concepts and confirm their practical applicability at the same time. Therefore, integrating remote labs with learning management systems can be quite beneficial from their utilization point of view.

3. Remote labs for physically disabled students: A lot of work has been doneto develop remote labs, mainly to provide access to equipment and experiments to students who are either located at remote places, or to those students who do not have any lab facilities available. However, there are still a section of underpriveldged students, with physical disabilities, who can neither work on physical nor on remote labs, due to their physical limitations. To cater to their special needs new methodologies need to be discovered.

\section{CONCLUSION}

In comparison with traditional e-contents (virtual Classrooms, e-projects) remote laboratory conception implies additional difficulties. These difficulties are due to teleoperation requirements providing touch and feel of a real lab. The user must be given the ownership of the hardware where he/she can design and execute an experiment of choice that includes all the pitfalls of real lab experiment i.e. making wrong wiring, debugging a circuit etc. In this work, we presented a model for analog communication remote lab to be used in traditional block diagram approach as well as device level approach that allows students to design, synthesize and evaluate their work.

\section{ACKNOWLEDGMENT}

The author C.M.Markan acknowledges Ministry of Human Resource and Development (MHRD) Government of India for the financial support under NMEICT Nationally Coordinated Project on Virtual Labs.

\section{REFERENCES}

[1] M.Llamas, M.Caeiro-Rodrfguez, and M.Castri, "User od ELearning Functionalities and Standards:The Spanish Case,'IEEE Trans.on Education, vol. 54,No. 4, November 2011.

[2] http://www-304.ibm.com/jct03001c/services/learning/ites.wss/us/ en?pageType $=$ page $\& \mathrm{c}=\mathrm{a} 0013864$
[3] J. Biggs, Teaching for Quality Learning at University. Maidenhead, U.K.: Open Univ. Press/McGraw-Hill, 2003.

[4] S. Ursulet and D. Gillet, "Introducing flexibility in traditional engineering education by providing dedicated on-line experimentation and tutoring resources", the International Conference onEngineering Education, Manchester, UK, August 18-21, 2002

[5] N. Ertugrul, "New area in engineering experiments: An integrated and interactive/learning approach, and real-time visualizations," Int. J. Eng.Educ., vol. 14, no. 5, pp. 344-355, 1998.

[6] C.Riman, E.Monacelli, I.Mougharbel, A.El-Hajj, "Remote Robotic Laboratory Experiment Platform Based on Tele-Programming", Communications in computer and information science, vol.293, pp 11-22

[7] Y.Bolea, A.Grau, "Green remote laboratory for chemical engineering degree: A new paradigm for training",Frontiers in education conference 2011

[8] M.T.Restivo, M.Joaquim, A.M.Lopes, C.M.Silva, F.Chouzal, “A remote laboratory in engineering measurement", IEEE Transactions on industrial electronics, vol 56 issue 12

[9] C.M.Markan, Priti Gupta, Manas, Goutam Kumar, Satendra Gupta, Scalable Multiuser Remote Laboratories provide on-demand hands-on laboratory experience, Remote Labs session, IEEE 2012 Conferenceon Technology and Society in Asia, Singapore Oct2729, 2012

[10] http://www.springerreference.com/docs/html/chapterdbid/320246. $\underline{\mathrm{html}}$

[11] Petros J. Axaopoulos, Konstantinos N. Moutsopoulosa \& Michael P. Theodoridis," Engineering Education Using a Remote Laboratory through the Internet", European Journal of Engineering Education, v37 n1 p39-48 2012

[12] Akram Abu-aisheh,Farid Farahmand,Implementation of a Remote Analog and Digital Communications Laboratory for eLearning, $10^{\text {th }}$ IEEE International Conference on Advanced Learning Technologies.

[13] Auer M., Pester A., Ursutiu D., and Samoila C., "Distributed Virtual and Remote Labs in Engineering”, Proc. of the ICIT'03 Intl. Conference on Industrial Technology, Maribor, Slovenia, December 10 - 12, 2003.

[14] Callaghan, MJ. Harkin, J., McGinnity, TM. and Maguire, LP.,"Paradigms in Remote Experimentation", iJOE(Intl.J. of Online Engg). ISSN: 1861-2121-Vol. 3, No. 4, Pg5-14, 2007

[15] Harvard V., Alamo J.D, et.al. "ilab: A scalable architecture for sharing onlineexperiments"., International Conf. on Engineering Education(ICEE2004), Gainesville, Florida, , Oct 16-21, 2004.

[16] Markan, CM., Bhatnagar, A., Gupta, A.," A Remote Triggered Electronics Laboratory using LXI methodology", Proc.of Intl. ConferenceonRemoteEngineering and Virtual Instrumentation, (REV-2010) Stockholm,June29-July 2, 2010.

[17] Multi-experiment Single Board Telecoms Trainer for the popularNI ELVISTM platform, Emona Telecoms Trainer ETT-202 HTTP://WWW.TIMS.COM

[18] Markan C.M., Gupta S., Mittal S.,Kumar G., "Remote Laboratories- A cloud based model for teleoperation of real laboratories", International Journal of Online Engineering (iJOE) 2013

[19] M.Tawfik, D.Lowe, S.Murray, M.D.1.Villefromoy, M.Diponio, E.Sancristobal, M.J.Albert, G.Diaz, M.Castro, "Grid Remote Laboratory Management System: Sahara Reaches Europe”, REV 2013

\section{AUTHORS}

Dr. C. M. Markan is an Associate Professor in Department of Physics and Computer Science, Dayalbagh Educational Institute, Dayalbagh, Agra, India. His areas of interests are in Neuromorphic and Adaptable Analog VLSI, Virtual and Remote Laboratories. He is a member of IEEE, Systems Society of India, and International Association of Online Engineering (cm.markan@gmail.com)

Goutam Kumar is a software developer in MHRD project in Dayalbagh Educational Institute, Dayalbagh, Agra, India. His interests are programming in java and web application development (goutamns@gmail.com). 


\section{SPECIAL FOCUS PAPER \\ REMOTE TRIGGERED ANALOG COMMUNICATION LABORATORY FOR E-LEARNING}

Sajal Mittal is a Graduate Student in Faculty of Electrical Engineering, Indian Institute of Technology, Kanpur. He did his under graduation from Electrical Department, Dayalbagh Educational Institute. He is student member of IEEE Society and actively participating in its activities (sajalmittal82@yahoo.co.in).

Priti Gupta is currently pursuing her phd from Dayalbagh educational institute. Her research areas include artificial neural networks, neuromorphic designs and quantum computing (gupta.priti.84@gmail.com).

Satendra Gupta is with the Department of Physics \& Computer Science, Dayalbagh Educational Institute, Agra, India (sunny.shal.gupta@gmail.com).
Anshul Satsangi is working as a graphic designer at Dayalbagh educational institute (satsangianshul@gmail.com).

Ashima Gupta is working as a web developer. She took her masters in computer applications from Dayalbagh educational institute (ashima4117@gmail.com).

Garima Kapur is pursuing her phd from Dayalbagh educational institute. Her interests include floating gate transistors and designing bio-inspired integrated circuits (kapur.garima@gmail.com).

This article is an extended and modified version of a paper presented at the EDUCON2013 conference held at Technische Universität Berlin, Berlin, Germany from March 13-15, 2013. Received 15 May 2013. Published as resubmitted by the authors 27 May 2013. 